\title{
Inhibitory control and its relationship to positivity biases in older adults
}

\author{
Caoimhe McManus \& David P. McGovern*
}

School of Psychology, Dublin City University, Glasnevin Campus, Dublin 9, Ireland

*Email: david.p.mcgovern@dcu.ie

\begin{abstract}
$\underline{\text { Abstract }}$
Older adults tend to focus on positive information over negative information; a phenomenon commonly referred to as the 'positivity effect'. Socioemotional Selectivity Theory posits that this effect stems from age-related shifts in goals and relies heavily on the active suppression of negative information. The current study tested the hypothesis that inhibitory control is a key determinant of positivity biases in older adults using anti-saccade and recognition memory tasks. Results indicated a significant correlation between levels of inhibitory control and the positivity effect. These findings highlight the key role played by inhibitory control in determining positivity biases amongst older adults.
\end{abstract}

Keywords: Positivity effect, aging, inhibitory control, cognitive control, emotional regulation 


\section{Introduction}

Aging is often associated with a decline in well-being due to reduced time left in life, deterioration of cognitive functioning and depreciation of physical health (Allard, 2010). Despite these well-known age-related decrements in physical and cognitive functioning, research has shown that positive well-being does not diminish, but may in fact improve with age (Carstensen \& DeLiema, 2018). Research in the psychology of aging has highlighted that older adults report higher levels of positive relative to negative affect (Orgeta, 2009), a decrease and levelling off of negative emotional experiences (Carstensen et al., 2000) and a more proficient capability of repairing negative mood states (Kliegel et al., 2007).

Recent research has also shown that there is an age-related shift in preference towards positive over negative information (Kennedy et al., 2004). For instance, compared to younger adults, older adults attend more to positive items and less to negative items leading to a recall bias for positive information (Isaacowitz et al., 2009), as well as an age-dependent bias towards positive attributes when making decisions (Kim et al., 2008). This age-related shift in the relative preference of positive over negative material in cognitive processing for older adults has been termed the 'positivity effect' (Kennedy et al., 2004) and has been replicated using a wide range of stimuli and experimental paradigms (see Reed et al., 2014 for metaanalysis).

The Socioemotional Selectivity Theory offers a potential explanation for the positivity effect (Carstensen, 1992), positing that there is a shift in goals in later life associated with the perception that time is more limited with advancing age. As such, emotionally-meaningful goals, such as maintaining close relationships, become more prominent in later life than information-seeking goals of early adulthood. In other words, while young adults assume that there is ample time left in life and focus on acquiring knowledge that may provide a future benefit, older adults perceive time to be limited and therefore focus on goals that will benefit 
them in the present rather than prioritizing goals associated with long-term rewards.

Therefore, positive emotional functioning in old age may be explained by motivational influences to maximise one's well-being in the here and now. In line with this theory, many age-related changes in cognition and behaviour are presumed to be the result of top-down influences associated with motivation rather than changes in the bottom-up processing of incoming information (Reed \& Carstensen, 2012).

According to this motivation-based explanation of the positivity effect, positivity stems from age-related shifts in goal priorities. Both selectively focusing on positive information and ignoring negative distracting information relies heavily on cognitive resources (Sasse et al., 2014) and it has been suggested that elderly people must employ cognitive control to pursue their emotional goals (Kryla-Lighthall \& Mather, 2009). In line with this assumption, research has shown that the positivity effect is evident in older adults when cognitive resources are relatively abundant and undivided (Joubert et al., 2018). In contrast to this, the positivity effect can be diminished, or even reversed, in older adults when attentional resources are exhausted and divided by a secondary task (Mather \& Knight, 2005). This reversal is explained by the fact that older adults are then unable to use their attentional resources for emotion regulation purposes (Brassen et al., 2011). Furthermore, it has been shown that older adults with lower degrees of executive control also show a diminished positivity effect (Mather \& Knight, 2005).

Despite the fact that control processes are regarded as a key determinant underlying age-related positivity biases, a recent meta-analysis on the positivity effect flagged the lack of consistent selection and reporting of background cognitive ability measures across studies in the field (Reed et al., 2014). It was recommended that future studies should incorporate common measures of cognitive control and to test this key tenet of the motivational perspective on the positivity effect. Additionally, it is unclear which aspect of cognitive 
control might facilitate the positivity effect in aging (Sasse et al., 2014). The unity/diversity framework of executive functioning identifies three related, but separable, executive functions: inhibition, updating and shifting (Miyake \& Friedman, 2012). Inhibition is used to select more appropriate behaviours that are consistent with one's goals in order to implement more adaptive goal-oriented behaviours (Miyake et al., 2000) and enable goals to regulate thought and action by suppressing otherwise automatic activation of goal-irrelevant information. Due to the fact that the positivity effect is believed to be the result of a shift of motivation in order to regulate emotions and ignore goal-irrelevant negative information, the relationship between inhibitory control and the positivity effect seems like the most appropriate candidate mechanism to investigate (see also Sakaki et al., 2019; Williams et al., 2020). Specifically, the current study hypothesised that older adults would show a bias towards remembering stimuli with a positive valence, in keeping with the previous literature, and additionally that older adults who had a higher (lower) level of inhibitory control would show a larger (smaller) positivity effect thereby drawing a connection between inhibitory control and the positivity effect. We also hypothesised that this bias towards positive information would be especially evident in the context of stimuli that depict events that are relevant to this age group (see e.g. Rhodes \& Anastasi, 2012).

\section{$\underline{\text { Methodology (1017 words) }}$}

\section{Participants}

Thirty younger (18-30 years: $\mathrm{M}=22.1, \mathrm{SD}=2.61,16$ male, 14 female) and 30 older $(60+$ years: $\mathrm{M}=64.76, \mathrm{SD}=5.12,17$ male, 13 female $)$ adults participated in the study. Criteria for inclusion in the study were: normal or corrected-to-normal vision; no previous history of neurological impairment; no previous diagnosis of dementia; and a minimum score of 25 on the Mini-Mental State Examination (MMSE). Younger participants were recruited from the student population of Dublin City University (DCU) and through a local sports club. 
Older participants were recruited via local retirement groups and sports clubs. All participants took part on a voluntary basis without reimbursement. All recruitment and experimental procedures were approved by the School of Psychology Research Ethics Committee, Dublin City University.

\section{$\underline{\text { Apparatus/Materials }}$}

Superlab 5.0 was used to present all aspects of the computer-based experiment and collect the resulting data. All participants completed the task on the researcher's personal laptop, which had a 14.5 inch screen running at a resolution of $1366 \times 768$ and a refresh rate of $60 \mathrm{~Hz}$.

\section{$\underline{\text { Procedure }}$}

The testing procedure was identical for younger and older participants and consisted of an initial viewing phase, an anti-saccade task (Hallet, 1978) and a recognition phase, where participants identified pictures they recognised from the viewing phase.

\section{Viewing Phase}

Stimuli for the viewing phase of this experiment consisted of 120 images, 112 of which were selected from the International Affective Picture System (IAPS; Lang et al., 2008 ) with the additional 8 images sourced from the internet. All pictures had a size of $600 \mathrm{x}$ 450 pixels and participants viewed the images from a distance of approximately $50 \mathrm{~cm}$. Pictures featured social situations, involving at least one person in a distinct positive, negative or neutral social situation. Half of the pictures depicted elderly people in social situations that were thematically more relevant for their respective age (e.g., playing with grandchildren, funeral, shopping etc.). The other half of the images showed younger people in social situations that were more typical for young adulthood (e.g., wedding, people in a 
brawl, two people running etc.). Affiliation of the images to the emotion and relevance categories was based on consensual classification by the research team.

The computer-based presentation of the images involved three images being presented simultaneously on a white background. In each of the 40 trials, two images were presented on the upper half of the screen and one in the centre of the lower half of the screen. Each trial contained one positive, one negative and one neutral image of the same age-category. Arrangement and order of the stimuli was pseudo-randomised such that each type of valence would occur equally often in the three positions. Furthermore, trials were arranged such that not more than three succeeding images belonged to the same age category. By presenting different emotional stimuli simultaneously, the need to engage in inhibitory control was maximised in order to selectively process goal-relevant information. Each trial started with a central fixation cross which was presented for a variable duration between 1000-3000 ms and the three images were subsequently displayed for $8000 \mathrm{~ms}$. Participants were instructed to look at the pictures as if they were watching them on TV, thus allowing participants to naturally focus their attention on goal-relevant information. After 20 trials of the task, a short break was provided to avoid lapses in attention. Four practice trials preceded the experiment to familiarise participants with the procedure and stimuli.

\section{Anti-Saccade Task}

After completion of the viewing phase of the paradigm, participants completed the anti-saccade task. In this task, participants were instructed to fixate on a centrally-presented cross for 1000-3000 ms. A cue, in the form of a black dot, was presented on either the left or right side of the screen for $100 \mathrm{~ms}$. As soon as this cue appeared, participants were instructed to shift their gaze to the opposite side of the screen where shortly after the cue (233 ms), an arrow was briefly presented for $33 \mathrm{~ms}$ (two video frames) followed by a mask. Participants 
were instructed to indicate the direction in which the arrow pointed (either left, right or up), by pressing one of three response keys. Twenty practice trials preceded the experiment to familiarise the participants with the task and stimuli. A total of 92 trials were presented in the main task with the number of correct responses recorded for each participant.

\section{Recognition Phase}

Following a short break after the anti-saccade task, participants completed the recognition phase of the study. In the recognition paradigm, the 120 stimuli presented in the viewing phase of the task were intermixed with 24 new stimuli which were selected from the IAPS. These 24 new stimuli contained 8 positive, 8 negative and 8 neutral images with equal number of old and young images in each valence category. In a self-paced manner, participants had to respond to each image individually and indicate whether they had seen it before by pressing the corresponding button: 'sure yes', 'probably yes', 'sure no', or 'probably no'. These response categories were offered to reduce response bias and to ensure participants remained engaged throughout. For analysis of the recognition data, sure and probable responses were collapsed into 'yes' and 'no' categories. The hit rate for each individual was then calculated by subtracting the number of false alarms from the number of correct hits. These values were computed for each stimulus category separately.

\section{Data Analysis}

Mixed-model ANOVAs were used to analyse the effects of emotion, image category and age group on the number of correct responses. All data entered into the ANOVAs were normally distributed and met the required assumptions. A one-tailed correlation was conducted between the number of correct responses on the anti-saccade task and number of positive items by older adults in the recognition phase of the study to assess the hypothesised relationship between cognitive control and the positivity effect. When multiple comparisons were performed, Bonferroni correction was used to maintain a family-wise Type 1 error rate 
at 0.05 and the adjusted p-value is reported. Effect sizes for significant $F$ and $t$ tests are represented by $\mathrm{h}_{\mathrm{p}}{ }^{2}$ and Cohen's $d$, respectively.

\section{$\underline{\text { Results }}$}

Younger adults outperform older adults on the anti-saccade task

To compare levels of inhibitory control between younger and older adults, participants completed an anti-saccade task in which they had to identify the orientation of a briefly presented arrow in a 3-alternative, forced-choice procedure. In line with previous studies (Abel \& Douglas, 2007; Bojko et al., 2004), current results showed that younger adults $(\mathrm{M}=86.03, \mathrm{SD}=4.28)$ correctly identified the direction of the arrow on more trials than their older counterparts $(\mathrm{M}=68.93, \mathrm{SD}=14.16)$ indicating higher levels of inhibitory control in the younger cohort $(t(58)=6.33, p<.001 ; d=1.63)$.

Older adults display a recall bias for stimuli depicting positive events

To assess participants' performance on the recognition memory task, participants were presented with a mixture of new and previously viewed stimuli, and were required to say whether or not the stimuli were presented during the viewing phase. A 3-way mixed ANOVA was conducted to determine the impact of stimulus valence (positive/negative/neutral), image category (own-age/otherage) and group (young/old) on hit rate. The predicted three-way interaction between emotion, image category and age group, was non-significant $\left(F(2,116)=1.52, p=.22, \mathrm{~h}_{\mathrm{p}}{ }^{2}=.03\right)$. This analysis also revealed no main effect of image category on the recall abilities of participants $(F(1,58)=.60, p=.44$, $\left.\mathrm{h}_{\mathrm{p}}{ }^{2}=.01\right)$; no interaction effect between image category and age $\left(F(1,58)=.002, p=.97, \mathrm{~h}_{\mathrm{p}}{ }^{2}<.001\right)$ and no interaction effect between image category and emotion $\left(F(2,116)=.12, p=.89, \mathrm{~h}_{\mathrm{p}}{ }^{2}=.002\right)$. For this reason, all subsequent analyses collapsed across the responses to the different image categories. These findings diverged from previous research (Rhodes \& Anastasi, 2012; Sasse et al., 2014) which suggested that memory enhancements tend to be especially apparent in the context of self-relevant stimuli (i.e., 'own-age' versus 'other-age' images). 
To assess whether the valence of the stimuli affected recall for the different age groups, a 2way factorial ANOVA was conducted with hit rate as the dependent variable and emotion and group as the independent variables. This analysis revealed a statistically significant main effect of emotion $\left(F(2,116)=33.93, p<.001, \mathrm{~h}_{\mathrm{p}}{ }^{2}=.37\right)$, such that negative items $(\mathrm{M}=25.38, \mathrm{SD}=0.74)$ were correctly identified more often than positive $(\mathrm{M}=24.73, \mathrm{SD}=0.68)$ and neutral items $(\mathrm{M}=20.58, \mathrm{SD}=0.74)$. There was also significant main effect of age group $\left(F(1,58)=12.75, p=.001, \mathrm{~h}_{\mathrm{p}}{ }^{2}=.18\right)$ whereby younger adults $(M=25.79, S D=0.88)$ correctly identified more items that older adults $(M=21.34$, $\mathrm{SD}=0.88$ ). Of particular relevance to the current study, there was a significant interaction effect between the valence of the recalled stimuli and age group $\left(F(2,116)=9.06, p<.001, \mathrm{~h}_{\mathrm{p}}{ }^{2}=.14\right)$, such that younger adults were more likely to remember stimuli with a negative valence while older adults recalled more stimuli with a positive valence (see Figure 1). On further examination of the interaction effect, post-hoc tests revealed that younger adults showed a general emotional memory enhancement for negative $(\mathrm{M}=29.07, \mathrm{SD}=6.06)$ and positive items $(\mathrm{M}=25.77, \mathrm{SD}=4.71)$, over neutral items $(\mathrm{M}=22.53, \mathrm{SD}=5.86)$ and also correctly identified more negative $(\mathrm{M}=29.07, \mathrm{SD}=6.06)$ than positive items $(\mathrm{M}=25.77, \mathrm{SD}=4.71)\left(\right.$ all $t \mathrm{~s}>3.61$, all $\left.p_{\text {adj }}<.05\right)$. On the other hand, older adults correctly identified more positive $(\mathrm{M}=23.70, \mathrm{SD}=5.77)$ than negative items $(\mathrm{M}=21.70, \mathrm{SD}=5.36)$, more negative than neutral items $(\mathrm{M}=18.63, \mathrm{SD}=5.67)$ and more positive than neutral items (all $t \mathrm{~s}>3.96$, all $\left.p_{\text {adj }}<.05\right)$. Together, these results provide further evidence for the idea that older adults are biased towards remembering stimuli that have positive valence.

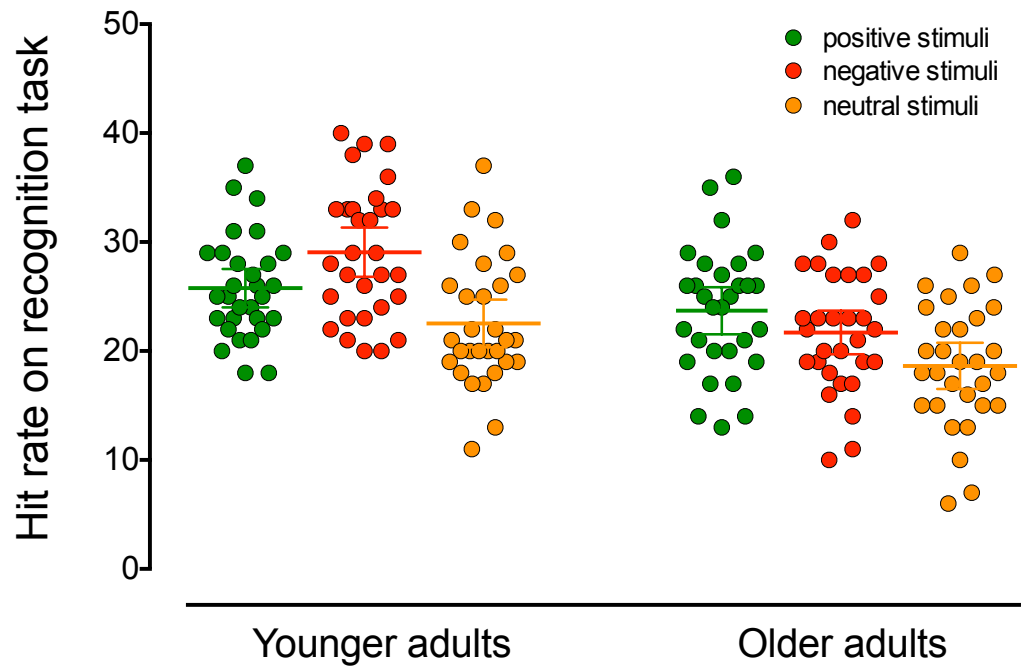

Figure 1: Hit rates for positive, negative and neutral stimuli on the recognition memory task. Individual data points represent the hit rate for one participant for one stimulus category. 
Higher levels of inhibitory control are associated with a larger positivity effect

To investigate the relationship between inhibitory control and the magnitude of the positivity effect in older adults, we plotted the number of correct responses on the anti-saccade task as a function of the number of correctly recalled positive items on the recognition memory task. Figure 2 shows this plot along with the best-fitting regression line to the datapoints. This scatterplot illustrates that there is a high degree of interindividual variability in the magnitude of both effects, but also a moderate correlation between hit rate on the anti-saccade task and the number of positive items identified by older adults $(r(30)=.35, p=.03$, one-tailed $)$, with levels of inhibitory control explaining approximately $11.9 \%$ of the variation in the recall scores of older adults. These findings suggest that higher levels of inhibitory control are associated with a larger positivity effect.

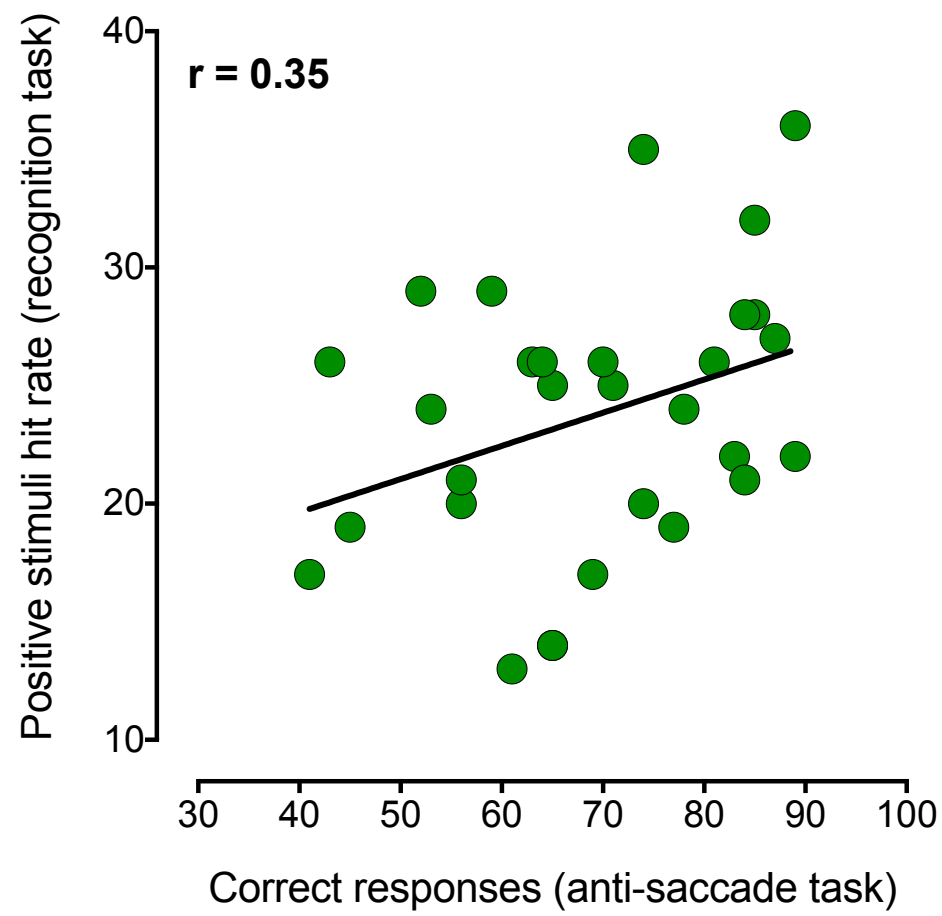

Figure 2: Scatterplot and best-fitting regression line to data from older adults on the recognition memory task and the anti-saccade task. There was a moderate correlation between the hit rate on the recognition memory task for positive stimuli and the number of correct responses on the anti-saccade task. 


\section{$\underline{\text { Discussion }}$}

Consistent with Socioemotional Selectivity Theory, this investigation found that the positivity effect in memory was evident amongst older adults. In addition, older adults who displayed higher levels of inhibitory control via the anti-saccade task also showed a stronger positivity effect in memory. These results suggest that the positivity effect in old age is determined not only by one's limited time perspective due to advancing age, but also by preserved cognitive control mechanisms which underlie emotional regulation.

Our findings highlight the potential link between inhibitory control and the positivity effect and provide support for motivational, top-down explanations of the positivity effect. Other models that attempt to explain the positivity effect include the 'aging brain' model, which posits that reduced responses to negative stimuli in older adults are a result of an agedependent decrease in neural activity in arousal-sensitive brain areas such as the amygdala (Cacioppo et al., 2011). This model draws on the argument that positive information is less cognitively demanding than negative information (Labouvie-Vief, 2003) thereby leading to an age-related positivity bias. However, our results, and similar findings in the field (Sakaki et al., 2019; Williams et al., 2020), argue against the aging brain model by suggesting that older adults who have preserved levels of inhibitory control demonstrate a larger positivity bias in memory. Taken together, these findings oppose the claim that positive information is preferred by older adults due to it being less cognitively demanding.

Another goal of the present study was to test a prediction as to whether observed biases for positive and negative items amongst older and younger adults respectively, were especially evident for stimuli of higher relevance for particular age groups. Based on the current results, it would appear that age-relevant stimuli do not lead to enhanced biases in emotional memory for younger or older adults, thus diverging from previous literature in the 
field which has emphasised the role of self-relevant stimuli (e.g. Denkinger \& Kinn, 2018; Rhodes \& Anastasi, 2012; Sasse et al., 2014).

While the current results are consistent with Socioemotional Selectivity Theory, it is important to acknowledge the limitations of the present study and address questions for future research. For example, due to the time constraints associated with the study, we did not incorporate all aspects of cognitive control into our study but instead focused on inhibitory control. Future studies should not only continue to test this facet of executive functioning in order to establish a rigorous body of research regarding its role in positivity biases, but should also include the remaining two facets of cognitive control (updating and shifting) into their paradigm. Furthermore, it must also be noted that, while there is emerging evidence highlighting the role of inhibitory control in determining positivity biases (see Sakaki et al., 2019; Williams et al., 2020), this study is the first of its kind to utilise the anti-saccade task in order to investigate this link.

It is also important to consider an overall criticism of the literature in this field which came to the fore during this investigation. One of the central findings of the current research is the age by valence interaction. As with most interactions, there are a number of ways to consider the effect. The current body of literature focuses on how older adults recall more positive information than younger adults; calling it a positivity effect emphasises this aspect of the interaction. However, another way to describe this effect is a negativity bias amongst younger adults, as they are more likely to recall negative information (Rozin \& Royzman, 2001). Some of the positivity effect in older adults may be due to goal-directed ignoring or suppression of negative information that helps to avoid a negativity effect rather than an enhancement of positive information. The extensive body of literature in this field is based on the premise that cognitive resources are key determinants of the positivity effect (Mather \& Knight, 2005; Reed \& Carstensen, 2012); however, as outlined in the recent meta-analysis by 
Reed et al., (2014), there is a lack of consistent selection and reporting of background cognitive ability measures across studies in the field. Therefore, the exact nature and role of cognitive control processes in this age by valence interaction remains unclear. Could the positivity effect actually be construed as a negativity effect which is controlled by older adults? This dual-process view of the positivity effect is an approach that has been recently investigated by Gronchi et al., (2018) who proffer a framework which suggests that the positivity effect amongst older adults is driven by two different processes: an automatic attention bias toward positive stimuli, and a controlled mechanism that diverts attention away from negative stimuli. This research suggests that older adults' enhancement of positive memories and impairment of negative memories are separable processes (Gronchi et al., 2018). Further investigations are warranted in order to investigate this framework which suggests the involvement of two distinct underlying neural mechanisms. In this respect, a neurally-informed modelling approach (O’Connell et al., 2018) could be a particularly fruitful avenue for future research and this approach has been successfully applied to field of perceptual decision-making to reveal distinct, nuanced age-related neural adaptations that would have been difficult to reveal using conventional methods (Dully et al., 2018; McGovern et al., 2018).

In sum, the findings of this investigation strengthen prior research which has indicated the presence of a positivity effect in older adults recall of emotionally evocative stimuli and highlights the key role of inhibitory control in determining positivity biases. These findings are consistent with the Socioemotional Selectivity Theory explanation of the positivity effect, but future research is warranted to elucidate the precise nature of the underlying cognitive processes involved in positivity biases amongst older adults.

\section{$\underline{\text { References }}$}


Allard, E. S. (2010). The influence of cognitive control effort on age-related positivity effects in visual fixation. (pp. 251-265). Brandeis University

Abel, L. A., \& Douglas, J. (2007). Effects of age on latency and error generation in internally mediated saccades. Neurobiology of Aging, 28(4), 627-637. https://doi.org/10.1016/j.neurobiolaging.2006.02.003

Bojko, A., Kramer, A. F., \& Peterson, M. S. (2004). Age Equivalence in Switch Costs for Prosaccade and Antisaccade Tasks. Psychology and Aging, 19(1), 226-234. https://doi.org/10.1037/0882-7974.19.1.226

Brassen, S., Gamer, M., \& Büchel, C. (2011). Anterior Cingulate Activation Is Related to a Positivity Bias and Emotional Stability in Successful Aging. Biological Psychiatry, 70(2), 131-137. https://doi.org/10.1016/j.biopsych.2010.10.013

Cacioppo, J. T., Berntson, G. G., Bechara, A., Tranel, D., \& Hawkley, L. C. (2011). Could an aging brain contribute to subjective well-being? The value added by a social neuroscience perspective. Social neuroscience: Toward understanding the underpinnings of the social mind, 249-262.

Carstensen, L. L. (1992). Social and emotional patterns in adulthood: support for socioemotional selectivity theory. Psychology and aging, 7(3), 331.

Carstensen, L. L., \& DeLiema, M. (2018). The positivity effect: A negativity bias in youth fades with age. Current Opinion in Behavioral Sciences, 19, 7-12. https://doi.org/10.1016/j.cobeha.2017.07.009

Carstensen, L. L., Mayr, U., Pasupathi, M., \& Nesselroade, J. R. (2000). Emotional Experience in Everyday Life Across the Adult Life Span. 12.

Denkinger, B., \& Kinn, M. (2018). Own-Age Bias and Positivity Effects in Facial Recognition. Experimental Aging Research, 44(5), 411-426. https://doi.org/10.1080/0361073X.2018.1521493 
Dully, J., McGovern, D. P., \& O'Connell, R. G. (2018). The impact of natural aging on computational and neural indices of perceptual decision making: A review. Behavioural brain research, 355, 48-55.

Gronchi, G., Righi, S., Pierguidi, L., Giovannelli, F., Murasecco, I., \& Viggiano, M. P. (2018). Automatic and controlled attentional orienting in the elderly: A dual-process view of the positivity effect. Acta Psychologica, 185, 229-234. https://doi.org/10.1016/j.actpsy.2018.02.008

Hallett, P. E. (1978). Primary and secondary saccades to goals defined by instructions. Vision research, 18(10), 1279-1296.

Isaacowitz, D. M., Allard, E. S., Murphy, N. A., \& Schlangel, M. (2009). The Time Course of Age-Related Preferences Toward Positive and Negative Stimuli. Journals of Gerontology: Series B, 64B(2), 188-192. https://doi.org/10.1093/geronb/gbn036

Joubert, C., Davidson, P. S. R., \& Chainay, H. (2018). When Do Older Adults Show a Positivity Effect in Emotional Memory? Experimental Aging Research, 44(5), 455468. https://doi.org/10.1080/0361073X.2018.1521498

Kennedy, Q., Mather, M., \& Carstensen, L. L. (2004). The Role of Motivation in the AgeRelated Positivity Effect in Autobiographical Memory. Psychological Science, 15(3), 208-214. https://doi.org/10.1111/j.0956-7976.2004.01503011.x

Kim, S., Healey, M. K., Goldstein, D., Hasher, L., \& Wiprzycka, U. J. (2008). Age differences in choice satisfaction: A positivity effect in decision making. Psychology and Aging, 23(1), 33-38. https://doi.org/10.1037/0882-7974.23.1.33

Kliegel M, Jäger T. Delayed-execute prospective memory performance: the effects of age and working memory. Dev Neuropsychol. 2006;30:819-843. doi: $10.1207 / \mathrm{s} 15326942 \mathrm{dn} 3003 \_4$. 
Kryla-Lighthall, N., \& Mather, M. (2009). The role of cognitive control in older adults' emotional well-being. In V. L. Bengston, D. Gans, N. M. Pulney, \& M. Silverstein (Eds.), Handbook of theories of aging (p. 323-344). Springer Publishing Company. Labouvie-Vief, G. (2003). Dynamic Integration: Affect, Cognition, and the Self in Adulthood. Current Directions in Psychological Science, 12(6), 201-206. https://doi.org/10.1046/j.0963-7214.2003.01262.x

Lang, P.J., Bradley, M.M., \& Cuthbert, B.N. (2008). International affective picture System (IAPS): Affective ratings of pictures and instruction manual. Technical Report A-8. University of Florida, Gainesville, FL

Mather, M., \& Knight, M. (2005). Goal-directed memory: The role of cognitive control in older adults' emotional memory. Psychology and Aging, 20(4), 554-570. https://doi.org/10.1037/0882-7974.20.4.554

McGovern, D. P., Hayes, A., Kelly, S. P., \& O’Connell, R. G. (2018). Reconciling agerelated changes in behavioural and neural indices of human perceptual decisionmaking. Nature human behaviour, 2(12), 955-966.

Miyake, A., \& Friedman, N. P. (2012). The nature and organization of individual differences in executive functions: Four general conclusions. Current directions in psychological science, 21(1), 8-14.

Miyake, A., Friedman, N. P., Emerson, M. J., Witzki, A. H., Howerter, A., \& Wager, T. D. (2000). The Unity and Diversity of Executive Functions and Their Contributions to Complex "Frontal Lobe" Tasks: A Latent Variable Analysis. Cognitive Psychology, 41(1), 49-100. https://doi.org/10.1006/cogp.1999.0734

O’Connell, R. G., Shadlen, M. N., Wong-Lin, K., \& Kelly, S. P. (2018). Bridging neural and computational viewpoints on perceptual decision-making. Trends in neurosciences, 41(11), 838-852. 
Orgeta, V. (2009). Specificity of age differences in emotion regulation. Aging \& Mental Health, 13(6), 818-826. https://doi.org/10.1080/13607860902989661

Reed, A. E., \& Carstensen, L. L. (2012). The Theory Behind the Age-Related Positivity Effect. Frontiers in Psychology, 3. https://doi.org/10.3389/fpsyg.2012.00339

Reed, A. E., Chan, L., \& Mikels, J. A. (2014). Meta-analysis of the age-related positivity effect: Age differences in preferences for positive over negative information. Psychology and Aging, 29(1), 1-15. https://doi.org/10.1037/a0035194

Rhodes, M. G., \& Anastasi, J. S. (2012). The own-age bias in face recognition: A metaanalytic and theoretical review. Psychological Bulletin, 138(1), 146-174. https://doi.org/10.1037/a0025750

Rozin, P., \& Royzman, E. B. (2001). Negativity bias, negativity dominance, and contagion. Personality and social psychology review, 5(4), 296-320.

Sakaki, M., Raw, J. A. L., Findlay, J., Thottam, M., Zwaan, R., \& Madan, C. (2019). Advanced Aging Enhances the Positivity Effect in Memory: Due to Cognitive Control or Age-Related Decline in Emotional Processing? Collabra: Psychology, 5(1). https://doi.org/10.1525/collabra.222

Sasse, L. K., Gamer, M., Büchel, C., \& Brassen, S. (2014). Selective Control of Attention Supports the Positivity Effect in Aging. PLoS ONE, 9(8), e104180. https://doi.org/10.1371/journal.pone.0104180

Williams, S. E., Lenze, E. J., \& Waring, J. D. (2020). Positive information facilitates response inhibition in older adults only when emotion is task-relevant. Cognition and Emotion, 1-14. https://doi.org/10.1080/02699931.2020.1793303 